\title{
OPTIMAL ASSET ALLOCATION IN LIFE INSURANCE: THE IMPACT OF REGULATION
}

BY

AN Chen AND Peter Hieber

\begin{abstract}
In a typical equity-linked life insurance contract, the insurance company is entitled to a share of return surpluses as compensation for the return guarantee granted to the policyholders. The set of possible contract terms might, however, be restricted by a regulatory default constraint - a fact that can force the two parties to initiate sub-optimal insurance contracts. We show that this effect can be mitigated if regulatory policy is more flexible. We suggest that the regulator implement a traffic light system where companies are forced to reduce the riskiness of their asset allocation in distress. In a utility-based framework, we show that the introduction of such a system can increase the benefits of the policyholder without deteriorating the benefits of the insurance company. At the same time, default probabilities (and thus solvency capital requirements) can be reduced.
\end{abstract}

\section{KEYWORDS}

Regulation, life insurance, default risk, utility maximization, risk sharing, multiobjective optimization.

\section{INTRODUCTION}

Equity-linked life insurance contracts usually provide a yearly or maturity guarantee for the policyholders. The insurance company is (up to some limits) liable for this return guarantee. As a compensation, the surplus above the guaranteed amount is shared between policyholders and the owners (equity holders) of the insurance company. Usually, the policyholders provide insurance premiums that are invested in a reference portfolio. The investment decision is, however, often affected by a regulatory default constraint. The regulator wants to restrict excessive risk taking of the insurance company (see, e.g., Gatzert and Schmeiser (2008); Dong et al. (2015); Filipović et al. (2015)). To restrict risk taking, the regulator can use a variety of tools: The regulator may enforce price constraints by 
introducing restrictions on premium calculation (see, e.g., MacMinn and Witt (1987)). Furthermore, the insurance company may be forced to provide riskbased capital as, for example, specified in the Solvency II accord. Risk-based capital regulation gains more and more importance in insurance regulation (see, e.g., European Parliament (2014)). The amount of risk capital needed is usually defined by imposing an upper bound on the insurance company's default probability (see, e.g., McCabe and Witt (1980)). If it is impossible to acquire new capital, this implicitly forces the insurer to restrict the riskiness of its investment decisions. The regulator may also directly impose restrictions on the insurance company's investment decision. In Germany, for example, pension funds and small insurance companies that are not subject to Solvency II rules need to bound the share of stocks and other risky investments in their portfolio. ${ }^{1}$

The introduction of (risk) constraints can, however, force the insurance company to offer Pareto inefficient contracts $^{2}$ (see, e.g., the detailed discussion in Rees et al. (1999)). The main research question in this paper is now how to mitigate this problem by designing a more flexible regulatory policy. The solution we suggest is a "traffic light system" that indicates whether the life insurance company is in danger of facing solvency problems (yellow signal) or even has severe and immediate problems (red signal). If the insurance company is in distress (yellow signal), the regulator enforces a decrease in the riskiness of the asset allocation. This traffic light solvency stress test is for example implemented in Denmark and Sweden, see, e.g., Jørgensen (2007) — similar ideas have been introduced in other European countries and in the Solvency II regulations. One advantage of the traffic light system is the fact that it is easy to implement and supervise.

We study the effect of the introduction of a "traffic light system" in a contingent claim approach. The stakes of policyholder and insurance company are options on future asset returns of the reference portfolio. The riskiness of this reference portfolio depends on the different traffic lights, and it is reduced as soon as the "yellow signal" appears. We introduce the regulatory optimization problem that determines optimal contract terms maximizing the policyholder's utility subject to a participation constraint of the insurance company (and possibly an additional regulatory default constraint). We assume that the insurance company is sufficiently supervised and cannot change the initially agreed investment strategy after contract initiation. This is in contrast to another strand of literature that examines the effect of "management discretion" or "risk-shifting" - where the insurance company benefits by departing from the contractually agreed strategy (at the expense of the policyholder). In our framework, we model default events of the insurance company continuously by a structural approach following the seminal paper of Black and Cox (1976). Comparing to the traditional regulatory scheme where the asset allocation is unchanged in distress, we show that the traffic light system increases the utility of the policyholder without deteriorating the utility of the equity holder.

In the past literature on equity-linked life insurance contracts, it is a common assumption that subsequent asset returns are independently and 
identically distributed. Typical asset models used are geometric Brownian motion or Merton jump-diffusion (see, e.g., Døskeland and Nordahl (2008); Gatzert and Schmeiser (2008); Schmeiser and Wagner (2013); Dong et al. (2015); Schlütter (2014); Filipović et al. (2015) and many others). Our flexible regulatory framework departs from this common assumption. The reference portfolio returns resulting from our investment strategy are non-Lévy and regime-dependent. Further complex and realistic models could be an interesting path for future research. ${ }^{3}$

The remainder of the paper is organized as follows. In Section 2, we describe the model setup and introduce the payoffs of the policy- and equity-holder, taking account of the possible default of the insurance company. More importantly, the flexible regulatory intervention (traffic light system) is presented. In the subsequent Section 3, the expected utility of the policyholder is computed analytically. In Section 4, this utility is maximized subject to a participation constraint of the insurance company. Thereby, we distinguish two cases: The insurance company can fully hedge the financial risks of the insurance contract (Section 4.1) or the insurance company is risk-averse and judges its payoffs according to a utility function (Section 4.2). In a numerical example, we then illustrate the advantage of the traffic light system. Finally, we provide some concluding remarks and an outlook for future research in Section 5 and detailed proofs in the Appendix.

\section{NOTATIONS AND MODEL SETUP}

Our model contains three parties: an insurance regulator, a representative shareholder (also equity holder) and a representative policyholder (also liability holder). The latter two constitute a life insurance company. We assume that the representative policyholder invests in an equity-linked life insurance contract with a maturity of $T$ years, $T<\infty$. At the initiation of the contract, the policyholder invests a lump sum $L_{0}$ in a single premium contract, the shareholder provides initial equity $E_{0}>0$. Consequently, the initial asset value $A_{0}$ of the insurance company is given by the sum of both contributions, i.e. $A_{0}:=L_{0}+E_{0}$. We denote the share of the policyholder's contribution (or equivalently the debt ratio of our insurance company) by $\alpha:=L_{0} / A_{0}$, where obviously $\alpha \in(0,1)$.

\subsection{Asset model and guaranteed amount}

Let us define a financial market consisting of one risk-free bond $B$ with riskfree interest rate $r$, i.e. $\mathrm{d} B_{t}=r B_{t} \mathrm{~d} t$ and $B_{0}=1$, and a risky asset which evolves according to

$$
\mathrm{d} S_{t}=\mu S_{t} \mathrm{~d} t+\sigma S_{t} \mathrm{~d} W_{t}, \quad S_{0}=1,
$$

where $\mu>r, \sigma>0$, and $W$ is a standard Brownian motion under the real world measure $\mathbb{P}$. To start with, we assume that the insurance company invests the total 
proceeds $A_{0}$ in a diversified portfolio of the risky and risk-free asset. Assume, a constant share $\theta_{1} \in[0,1]$ is invested in the risky asset $S$ and the remainder in the risk-free asset $B$. With the initial asset investment $A_{0}>0$, this yields the following asset dynamics:

$$
\mathrm{d} A_{t}=\left(r+\theta_{1}(\mu-r)\right) A_{t} \mathrm{~d} t+\sigma \theta_{1} A_{t} \mathrm{~d} W_{t} .
$$

The asset dynamics remains a log-normal process with a volatility of $\sigma \theta_{1}$. The amount guaranteed to the policyholder at time $t \in[0, T]$ is assumed to be $L_{t}=$ $L_{0} e^{g t}$, where $g \leq r$ is the guaranteed rate. We want to analyze the financial risks of equity-linked insurance contracts, therefore, as is common in this context (see, e.g., Bauer et al. (2005); Døskeland and Nordahl (2008)), we purely consider financial risks and ignore mortality risk.

\subsection{Default of the insurance company}

We want to explicitly take the default risk of the insurance company into account. Therefore, we make use of a structural approach and assume that the insurance company defaults as soon as its assets $A_{t}$ hit or drop below a specified percentage $\eta$ of the guaranteed amount $L_{t}$. Thus, we introduce a default barrier $D_{t}:=\eta L_{0} e^{g t}$ whose accrual rate $g$ is the same as for the guaranteed amount. The time of default is then the first-hitting time $\tau$ defined by

$$
\tau:=\inf \left\{t \geq 0 \mid A_{t} \leq D_{t}\right\},
$$

where we set $\inf \{\emptyset\}=\infty$. The default parameter $\eta$ is assumed to be smaller than $A_{0} / L_{0}$ such that the company is solvent initially.

\subsection{Terminal payoff to liability and equity holder}

The insurance payoff to the policyholder is contingent on whether the insurance company survives the maturity date $T$. If there is no premature default of the insurance company, the policyholder receives the following terminal payoff: ${ }^{4}$

$$
\begin{aligned}
\Psi_{L}\left(A_{T}\right) & := \begin{cases}A_{T} & \text { if } A_{T} \leq L_{T} \\
L_{T}+\delta\left[\alpha A_{T}-L_{T}\right]^{+} & \text {else }\end{cases} \\
& =L_{T}+\delta\left[\alpha A_{T}-L_{T}\right]^{+}-\left[L_{T}-A_{T}\right]^{+},
\end{aligned}
$$

where we denote by $[\cdot]^{+}$the maximum $\max \{\cdot, 0\}$. The participation rate $\delta \in$ $[0,1]$ is the percentage of surpluses that is credited to the liability holder. If there is no premature default, the terminal contract payoff is a combination of a fixed payment $L_{T}$, a bonus call and a shorted put option on the insurance company's assets. The shorted put option refers to losses of the liability holder if the company is not defaulted prematurely but assets at maturity are insufficient to cover the guaranteed amount. 


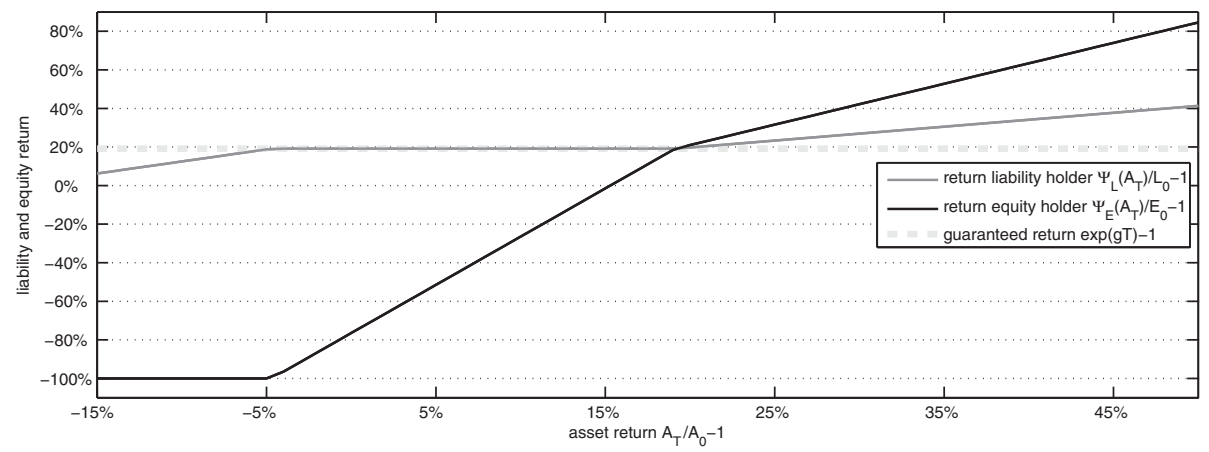

FIGURE 1: Return of liability and equity holder dependent on the asset return $A_{T} / A_{0}-1$ in case that the insurance company survives until maturity $T$, i.e. $\tau>T$. The parameters are set as $A_{0}=1, L_{0}=\alpha A_{0}=0.8$, $D_{0}=0.85, \delta=0.72, g=1.75 \%, T=10, \mu=6 \%, r=2.5 \%, \theta_{1}=0.2$, and $\sigma=0.2$.

In the case of premature default, a rebate payment is provided to the policyholder at time $\tau$. This rebate payment is given by the minimum of the current asset value $A_{\tau}=D_{\tau}$ and the current liabilities $L_{\tau}: \Xi_{L}(\tau):=\min \left(L_{\tau}, D_{\tau}\right)$. If we - for time consistency reasons - assume that the rebate payment is until time $T$ accumulated at the risk-free rate $r$, the policyholder receives the following contract payoff at time $T$ :

$$
V_{L}\left(A_{T}\right):=\mathbb{1}_{\{\tau>T\}} \Psi_{L}\left(A_{T}\right)+\mathbb{1}_{\{\tau \leq T\}} e^{r(T-\tau)} \Xi_{L}(\tau),
$$

where $\mathbb{1}_{\{B\}}$ is an indicator function which gives 1 if $B$ occurs and 0 otherwise. The equity holder always obtains the residual asset value. If there is no premature default of the insurance company, the payoff to the equity holder is

$$
\begin{aligned}
\Psi_{E}\left(A_{T}\right):=A_{T}-\Psi_{L}\left(A_{T}\right) & = \begin{cases}0 & \text { if } A_{T} \leq L_{T} \\
A_{T}-L_{T} & \text { if } L_{T}<A_{T} \leq A_{0} e^{g T} \\
A_{T}-L_{T}-\delta\left[\alpha A_{T}-L_{T}\right]^{+} & \text {else }\end{cases} \\
& =\left[A_{T}-L_{T}\right]^{+}-\delta\left[\alpha A_{T}-L_{T}\right]^{+} .
\end{aligned}
$$

If there is premature default, a rebate payoff $\Xi_{E}(\tau):=D_{\tau}-\min \left(L_{\tau}, D_{\tau}\right)$ is provided to the equity holder. More compactly, the total payoff to the equity holder at maturity $T$ is thus given by

$$
V_{E}\left(A_{T}\right):=\mathbb{1}_{\{\tau>T\}} \Psi_{E}\left(A_{T}\right)+\mathbb{1}_{\{\tau \leq T\}} e^{r(T-\tau)} \Xi_{E}(\tau) .
$$

Hereby, we have again accrued the rebate payment at time $\tau$ with the risk-free rate until the maturity date.

If the company survives maturity $T$, the payoff to the liability and equity holder are given in Equations (4), and (6), respectively. A numerical example, presenting the maturity return of liability and equity holder dependent on the asset return $A_{T} / A_{0}-1$, is given in Figure 1. The dashed line corresponds to 
the guaranteed return $e^{g T}-1$. If the asset return is greater than the guaranteed return, the liability holder (grey line) receives a bonus payment. If the insurance company survives the maturity, but the assets at that time are insufficient to cover the liabilities, the liability holder receives a return less than the guaranteed one. From Figure 1, one can observe that the equity holder's return (black line) is much more volatile than the liability holder's return.

\subsection{Payoff evaluation}

We now assume that the liability holder judges the final payoff according to a utility function, see Definition 2.1.

Definition 2.1 (Utility function: Inada conditions). The utility function $u_{L}(x)$ is twice differentiable on $\mathbb{R}^{+}$, is strictly increasing in $x$, i.e. $u_{L}^{\prime}(x)>0$, is concave, i.e. $u_{L}^{\prime \prime}(x)<0$, and has the following limiting derivatives: $\lim _{x \rightarrow 0} u_{L}^{\prime}(x)=\infty$ and $\lim _{x \rightarrow \infty} u_{L}^{\prime}(x)=0$.

Later on, we exemplarily use power utility, see Example 2.2.

Example 2.2 (Power utility). Let $\gamma_{L}>0, \gamma_{L} \neq 1$ be the relative risk-aversion parameter, then the power utility function is given by $u_{L}\left(V_{L}\right):=V_{L}^{1-\gamma_{L}} /\left(1-\gamma_{L}\right)$.

The liability holder evaluates the payment streams according to the objective function

$$
\mathcal{U}_{L}(\Theta):=\mathbb{E}_{\mathbb{P}}\left[u_{L}\left(V_{L}\left(A_{T}\right)\right)\right],
$$

where the parameter vector $\Theta$ contains the control variables, i.e. the participation rate and the asset allocation parameters. ${ }^{5}$ For interpretation purposes, we later use certainty equivalents defined as $C E_{L}(\Theta):=\left(\left(1-\gamma_{L}\right) \mathcal{U}_{L}(\Theta)\right)^{1 /\left(1-\gamma_{L}\right)}$.

\subsection{Regulatory intervention}

The aim of this paper is to examine the impact of regulation on the contract terms. Under Solvency II, solvency capital requirements are determined according to the $99.5 \%$ Value-at-Risk over a one-year time horizon (see, for example Art. 101 of the Solvency II directive). This approach restricts the annual default probability of the insurance company to $0.5 \%$. To keep our setup analytically tractable, we do not bound annual default probabilities but instead assume that the regulator sets an upper bound $\epsilon$ on the contract's default probability $\mathbb{P}(\tau \leq T)$. Then, we distinguish two cases:

(1) Traditional regulatory framework: In this setup, the asset portfolio follows dynamics (2), i.e. the portfolio's returns have a constant volatility. We choose the term "traditional" as this is the setup that is frequently used in related 
literature, see, among others, Grosen and Jørgensen (2002), Chen and Suchanecki (2007) and Schmeiser and Wagner (2015). In this setup, it is possible to show that the default constraint is equivalent to a bound on the share of risky assets $\theta_{1}$, i.e. $0 \leq \theta_{1} \leq \theta^{\max }$ for a given constant $\theta^{\max } \leq 1$. $^{6}$

If, however, under Solvency II, capital requirements are determined annually according to a $99.5 \%$ Value-at-Risk, this would allow the insurer to start with an initially higher investment in risky assets. If the financial situation weakens in the coming years, risk can be reduced to be still in line with solvency capital requirements. This motivates our second framework:

(2) Flexible regulatory framework: Here, the regulator allows for flexibility in the investment strategy, while still keeping the default probability constraint $\mathbb{P}(\tau \leq T) \leq \epsilon$. The concept is in analogy to Solvency II regulations in Europe where the regulator has the possibility to intervene, as soon as the assets drop below some critical level $\left\{K_{t}\right\}_{t \geq 0}$ (yellow signal) to avoid a default event. If the company's assets nevertheless drop below the default barrier $\left\{D_{t}\right\}_{t \geq 0}$ (red signal), the insurance company defaults. The possible interaction in case of the "yellow signal" gives more freedom to act in the interests of both liability and equity holder.

We now need some more technical details for the flexible regulatory framework. The second (upper) regulatory threshold $K$ is set as

$$
K_{t}:=K_{0} e^{g t},
$$

where $D_{0}=\eta L_{0}<K_{0}<A_{0}$. The hitting time of this barrier is denoted by

$$
\hat{\tau}:=\inf \left\{t \geq 0 \mid A_{t} \leq K_{t}\right\},
$$

where we again set $\inf \{\emptyset\}=\infty$. In case this barrier is hit, the regulator may once force the insurance company to change its investment strategy from $\theta_{1}$ to $\theta_{2} \in[0,1]$. Then, the asset value process is - for $t \geq 0$ - given by

$$
\mathrm{d} A_{t}=\left(r+\theta_{Z_{t}}(\mu-r)\right) A_{t} \mathrm{~d} t+\theta_{Z_{t}} \sigma A_{t} \mathrm{~d} W_{t}, \quad A_{0}>0,
$$

where $Z_{t}=1$ for $t \leq \hat{\tau}$ and $Z_{t}=2$ for $t>\hat{\tau}$. The effect of this more flexible design on the benefits of equity and liability holder is analyzed in the remainder of this paper. For reasons of analytical tractability, we do not consider a strategy recovery of the insurance company, i.e. it is not possible to return to the original asset strategy $\theta_{1}$.?

Under this more flexible regulation, the default-triggering event remains unchanged. A default occurs when the asset process $A_{t}$ hits the lower threshold $D_{t}$ (i.e. if $\tau \leq T$ ). Since the asset process is continuous and the regulatory barrier $K_{t}$ by definition greater than $D_{t}$, the event $\{\tau \leq T\}$ implies that $\{\hat{\tau} \leq T\}$, i.e. the upper threshold is hit before the lower one. ${ }^{8}$ 


\section{THEORETICAL RESULTS}

In order to determine the optimal investment strategy and examine the regulatory effects on it, we need to compute the expected utility of the policyholder (8).

\subsection{Traditional regulatory framework}

In the first case, we assume that there is no regulatory barrier $\left\{K_{t}\right\}_{t \geq 0}$ and thus the investment strategy stays constant at $\theta_{1} \in[0,1]$. Theorem 3.1 gives analytical expressions for the expected utility of the terminal payoff to the policyholder.

Theorem 3.1 (Expected utility: Traditional regulatory framework). Assume the model setup as described in Section 2 with asset process (2). Then, the desired expectation is given by

$$
\mathcal{U}_{L}(\Theta)=: \kappa_{L}^{(1)}\left(A_{0}, D_{0}, L_{0}, T\right),
$$

where $\kappa_{L}^{(i)}(\cdot), i=1,2$, can be computed via

$$
\begin{aligned}
\kappa_{L}^{(i)}\left(A_{0}, D_{0}, L_{0}, T\right)=\int_{0}^{T} & u_{L}\left(e^{r(T-\tau)} \Xi_{L}(\tau)\right) \cdot f^{(i)}\left(0, \tau, A_{t}, D_{t}\right) d \tau \\
& +\int_{\ln \left(\frac{D_{0}}{A_{0}}\right)}^{\infty} u_{L}\left(\Psi_{L}(\tau)\right) \cdot g^{(i)}\left(y, 0, T, A_{0}, D_{0}\right) d y,
\end{aligned}
$$

where $g$, respectively $f$, is for $A_{t}>D_{t}, \tau>T, y>\ln \left(D_{t} / A_{t}\right)$, defined as

$$
\begin{aligned}
g^{(i)}\left(y, t, T, A_{t}, D_{t}\right) & :=\frac{1}{\sigma \theta_{i} \sqrt{T-t}} \varphi\left(\frac{y-\tilde{\mu}_{i}(T-t)}{\sigma \theta_{i} \sqrt{T-t}}\right)\left(1-e^{-\frac{\ln \left(D_{t} / A_{t}\right)^{2}-y \ln \left(D_{t} / A_{t}\right)}{\sigma^{2} \theta_{i}^{2}(T-t)}}\right), \\
f^{(i)}\left(t, \tau, A_{t}, D_{t}\right) & :=\frac{-\ln \left(D_{t} / A_{t}\right)}{\sigma \theta_{i}(\tau-t)^{\frac{3}{2}}} \varphi\left(\frac{\ln \left(D_{t} / A_{t}\right)-\tilde{\mu}_{i}(\tau-t)}{\sigma \theta_{i} \sqrt{\tau-t}}\right), \\
\tilde{\mu}_{i} & :=r+\theta_{i}(\mu-r)-g-\sigma^{2} \theta_{i}^{2} / 2,
\end{aligned}
$$

and $\varphi(\cdot)$ denotes the density of the standard normal distribution.

Proof. See the Appendix.

In the case of power utility, most of the integrals in Theorem 3.1 can be derived analytically. Apart from the expected utility, one can also derive the default probability analytically. On the time interval $[0, T]$, it is given by

$$
\mathbb{P}(\tau \leq T)=\Phi\left(\frac{\ln \left(D_{0} / A_{0}\right)-\tilde{\mu}_{1} T}{\sigma \theta_{1} \sqrt{T}}\right)+\left(\frac{D_{0}}{A_{0}}\right)^{\frac{2 \tilde{\mu}_{1}}{\sigma^{2} \theta_{1}^{2}}} \Phi\left(\frac{\ln \left(D_{0} / A_{0}\right)+\tilde{\mu}_{1} T}{\sigma \theta_{1} \sqrt{T}}\right),
$$

where $\Phi(\cdot)$ is the standard normal distribution function and $\tilde{\mu_{1}}$ is defined as in Theorem 3.1, see also the Appendix. ${ }^{9}$ 


\subsection{Flexible regulatory framework}

Now, we are going to derive the same results as in Theorem 3.1 under the assumption that the investment strategy is changed from $\theta_{1}$ to $\theta_{2}$ as soon as the regulatory barrier $\left\{K_{t}\right\}_{t \geq 0}$ is hit. This leads to the asset process given by (11). Technically, this setup is still analytically tractable: Until first hitting the regulatory threshold $K$ at time $\hat{\tau}$, the asset process behaves as a geometric Brownian motion - one of the rare cases where the first-hitting time density is known analytically (the hitting time is distributed according to an inverse Gaussian law, see, for example, Folks and Chhikara (1978)). At time $\hat{\tau}$, the asset value equals the barrier $K_{\hat{\tau}}$. After this hitting time, the assets are again a geometric Brownian motion now with a different mean and volatility parameter due to the changed investment strategy $\theta_{2}$. Thus, the time to default follows again an inverse Gaussian law. To sum up, the default time $\tau$ is given by the convolution of two inverse Gaussian random variables. The default probability can be evaluated via

$$
\begin{aligned}
\mathbb{P}(\tau \leq T) & =\int_{0}^{T} \mathbb{P}\left(\tau \leq T \mid A_{\hat{\tau}}=K_{\hat{\tau}}\right) \cdot f^{(1)}\left(0, \hat{\tau}, A_{0}, K_{0}\right) \mathrm{d} \hat{\tau} \\
& =\int_{0}^{T} \int_{0}^{T-\tau} f^{(2)}\left(\hat{\tau}, \tau, K_{\hat{\tau}}, D_{\hat{\tau}}\right) \cdot f^{(1)}\left(0, \hat{\tau}, A_{0}, K_{0}\right) \mathrm{d} \hat{\tau} \mathrm{d} \tau,
\end{aligned}
$$

with $f$ as defined in Theorem 3.1. Equation (12) results as the special case $\theta_{1}=\theta_{2}$. Similarly to Theorem 3.1, one can derive the expected utility $\mathbb{E}_{\mathbb{P}}\left[u_{L}\left(V_{L}\left(A_{T}\right)\right)\right]$ of the liability holder, see Theorem 3.2.

Theorem 3.2 (Expected utility: Flexible regulatory framework). Assume the model setup as described in Section 2 with asset process (11). The regulator may intervene at time $\hat{\tau}$ - the first-hitting time of the insurance company's assets $A$ breaching the regulatory barrier $K_{t}=K_{0} e^{g t}$. At time $\hat{\tau}$, the insurance company is forced to change its investment strategy from $\theta_{1}$ to $\theta_{2}$. Then, the desired expectation is given by

$$
\mathcal{U}_{L}(\Theta)=: \zeta_{L}\left(A_{0}, D_{0}, K_{0}, L_{0}, T\right),
$$

where

$$
\begin{aligned}
\zeta_{L}\left(A_{0}, D_{0}, K_{0}, L_{0}, T\right)= & \int_{0}^{T} \kappa_{L}^{(2)}\left(K_{\hat{\tau}}, D_{\hat{\tau}}, L_{\hat{\tau}}, \hat{\tau}, T\right) \cdot f^{(1)}\left(0, \hat{\tau}, A_{0}, K_{0}\right) \mathrm{d} \hat{\tau} \\
& +\int_{\ln \left(K_{0} / A_{0}\right)}^{\infty} u_{L}\left(\Psi_{L}\left(A_{0} e^{y+g T}\right)\right) \cdot g^{(1)}\left(y, 0, T, A_{0}, K_{0}\right) \mathrm{d} y,
\end{aligned}
$$

with $f, g$, and $\kappa_{L}$ as defined in Theorem 3.1.

Proof. See the Appendix.

Remark 3.3 (Implementation of Theorems 3.1 and 3.2). The expectations presented in Theorems 3.1 and 3.2 are integrals over normal densities. Thus, they can easily be implemented at high precision. Computation time is within fractions 
of seconds. That is why, it does not make sense to further simplify the given expressions and solve the integrals analytically, although it is, for example, possible to present $\kappa_{L}^{(1)}\left(A_{0}, D_{0}, L_{0}, T\right)$ in Theorem 3.1 in a (lengthy) closed-form expression.

\section{OPTIMAL CONTRACT TERMS}

We have now analytic expressions for the utility of the policyholder. In this section, we use these results to determine optimal contract terms.

\subsection{Financial risk can be hedged}

First, we want to consider a complete market setting where the policy risk can be hedged on financial markets (see, e.g., Brennan and Schwartz (1976); Døskeland and Nordahl (2008)). If there is no arbitrage, the hedging portfolio determines a unique fair price of the insurance contract. Therefore, one changes the dynamics of the reference portfolio from the market probability measure $\mathbb{P}$ to the (unique) equivalent pricing measure $\mathbb{Q}$ with the risk-free bond $\mathrm{d} B_{t} / B_{t}=r \mathrm{~d} t$ as reference asset. Under $\mathbb{Q}$, the risky asset evolves as

$$
\mathrm{d} S_{t}=r S_{t} \mathrm{~d} t+\sigma S_{t} \mathrm{~d} W_{t}^{\mathbb{Q}},
$$

where still $B_{0}=S_{0}=1$ and $W^{\mathbb{Q}}$ is a standard Brownian motion under $\mathbb{Q}$. If an insurance contract is fairly priced, the expected discounted payoff to the insurance company under $\mathbb{Q}$ equals its arbitrage-free initial stake $(1-\alpha) A_{0}$, i.e.

$$
\begin{aligned}
(1-\alpha) A_{0} & =E_{0}=\mathbb{E}_{\mathbb{Q}}\left[e^{-r T} V_{E}\left(A_{T}\right)\right] \\
& =\mathbb{E}_{\mathbb{Q}}\left[\mathbb{1}_{\{\tau>T\}} e^{-r T} \Psi_{E}\left(A_{T}\right)+\mathbb{1}_{\{\tau \leq T\}} e^{-r \tau} \Xi_{E}(\tau)\right] .
\end{aligned}
$$

Similarly (and equivalently ${ }^{10}$ ) from the policyholder's viewpoint, one has to ensure that

$$
\alpha A_{0}=L_{0}=\mathbb{E}_{\mathbb{Q}}\left[e^{-r T} V_{L}\left(A_{T}\right)\right]=\mathbb{E}_{\mathbb{Q}}\left[\mathbb{1}_{\{\tau>T\}} e^{-r T} \Psi_{L}\left(A_{T}\right)+\mathbb{1}_{\{\tau \leq T\}} e^{-r \tau} \Xi_{L}(\tau)\right],
$$

with $V_{E}\left(A_{T}\right)$ and $V_{L}\left(A_{T}\right)$ as defined in Equations (7) and (5), respectively. In this setup, the equity holder initiates contracts if and only if those contracts are at least fairly priced, i.e. if $E_{0} \leq \mathbb{E}_{\mathbb{Q}}\left[e^{-r T} V_{E}\left(A_{T}\right)\right]$. We understand this inequality as the participation constraint of the equity holder. Contracts are initiated if and only if the market value of the contract payoff is higher or equal to the initial investment $E_{0}$. As we have discussed earlier, the policyholder judges contract terms according to the utility function $\mathcal{U}_{L}(\Theta)$. The parameters $\Theta=\left(\delta, \theta_{1}\right)$ in the traditional regulatory framework and $\Theta=\left(\delta, \theta_{1}, \theta_{2}\right)$ under the flexible regulatory framework can be influenced by the two parties when signing the 
insurance contract. We now set the regulatory optimization problem to determine the optimal parameter vector $\Theta$. Therefore, the policyholder's utility is maximized subject to the participation constraint of the equity holder, i.e.

$$
\begin{aligned}
& \max _{\Theta} \mathcal{U}_{L}(\Theta) \\
& \text { s.t. } E_{0} \leq \mathbb{E}_{\mathbb{Q}}\left[e^{-r T} V_{E}\left(A_{T}\right)\right],
\end{aligned}
$$

see also (1) in Filipović et al. (2015). Recall that we assume that the parameter vector $\Theta=\left(\delta, \theta_{1}\right)$, respectively $\Theta=\left(\delta, \theta_{1}, \theta_{2}\right)$, is agreed at contract initiation and left unchanged until the maturity of the contract. However, this contract decision might be further influenced by an additional regulatory constraint, for example imposing a default constraint. In this case, the regulatory optimization problem has to be modified to

$$
\begin{array}{ll}
\max _{\Theta} & \mathcal{U}_{L}(\Theta) \\
\text { s.t. } & E_{0} \leq \mathbb{E}_{\mathbb{Q}}\left[e^{-r T} V_{E}\left(A_{T}\right)\right] \\
& \mathbb{P}(\tau \leq T) \leq \epsilon,
\end{array}
$$

where $\epsilon>0$ is the upper bound on the company's default probability. Due to the non-linearity of participation and default constraint, problems (17) and (18) cannot be solved explicitly. However, one can show that in the optimal solution, the participation constraint is binding. ${ }^{11}$ In the following, we solve optimization problems (17) and (18) numerically.

4.1.1. Numerical illustrations. We now illustrate optimization problem (17) in a numerical example and discuss the effect of the flexible regulatory scheme as opposed to the traditional regulatory scheme. In contrast to the asset allocation strategy, parameters like $D_{0}, \eta$, are either set or restricted by national law (insolvency condition $\eta$ ) or changing them might be difficult for the insurance company (equity share $1-\alpha$ ):

- In line with the balance sheet data from big German insurance companies where equity is mainly composed of stocks and undistributed reserves, we set the equity share to $1-\alpha=5 \%$. From this, the initial assets are (without loss of generality) $A_{0}=100$; the initial single premium is $L_{0}=95$.

- We limit a possible underfunding of the insurance company. Recall that the company defaults as soon as the assets drop below the default barrier $D_{t}=$ $D_{0} e^{g t}$. We distinguish the two cases where a slight underfunding $\left(D_{0}=94\right.$, $\eta=94 / 95 \approx 0.99)$ or a rather high underfunding $\left(D_{0}=90, \eta=90 / 95 \approx\right.$ $0.95)$ is allowed.

- The utility function of the policyholder is assumed to be power utility with a relative risk-aversion parameter $\gamma_{L}=3$. 
TABLE 1

RESULTS OF THE UTILITY MAXIMIZATION PROBLEMS (17) (NO DEFAULT CONSTRAINT) AND (18) (DEFAULT CONSTRAINT $P D \leq 0.5 \%$ ) IN THE TRADITIONAL (TOP, CONSTANT-MIX, $\theta_{1}=\theta_{2}$ ) AND THE FLEXIBLE (BOTTOM) REGULATORY FRAMEWORK . THE ANNUALIZED DEFAULT PROBABILITY P $D$ IS DEFINED AS $P D:=1-(1-\mathbb{P}(\tau \leq T))^{1 / T}$. THE RESTRICTION $P D \leq 0.5 \%$ IS EQUIVALENT TO $\epsilon=1-(1-0.5 \%)^{T} \approx 0.049 \mathrm{IN}(18)$.

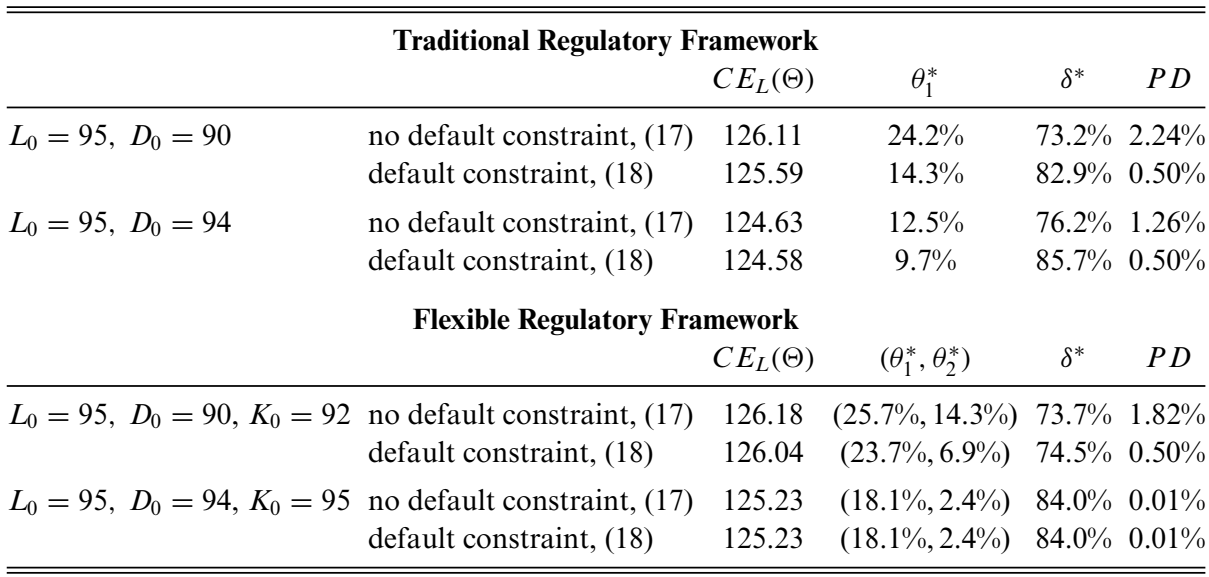

Further, we set the time horizon to $T=10$ and the financial market parameters to $\mu=6 \%, r=2.5 \%$ and $\sigma=0.2$. The guaranteed rate is set to $g=2.0 \%$. The qualitative results in this section are consistent if the parameters $\alpha, \eta, \gamma_{L}, T, \mu, r, \sigma, g$ are changed in a reasonable way.

In the following, we want to solve the utility maximization problems without default constraint (17) and with default constraint (18) in the traditional regulatory framework, see Table 1 (top) for two different parameter sets differing by the default barrier $D_{0}=90$, respectively $D_{0}=94$. In Table 1 , we display the optimal certainty equivalent $C E_{L}(\Theta):=\left(\left(1-\gamma_{L}\right) \mathcal{U}_{L}(\Theta)\right)^{1 /\left(1-\gamma_{L}\right)}$ of the policyholder. We find that the regulatory restriction on the annualized default probability $P D,{ }^{12}$ i.e. $P D \leq 0.5 \%$, forces to decrease the riskiness of the investment strategy from an equity share of $\theta_{1}^{*}=24.2 \%$ to $\theta_{1}^{*}=14.3 \%$ (if $D_{0}=90$ ), respectively from $\theta_{1}^{*}=12.5 \%$ to $\theta_{1}^{*}=9.7 \%$ (if $D_{0}=94$ ). Such a decrease has two effects on the policyholder's contract value of (5): It reduces the value of the bonus option $\left[\alpha A_{T}-L_{T}\right]^{+}$in (4) but reduces default probabilities and increases the value of the short put option $-\left[L_{T}-A_{T}\right]^{+}$in (4). The default constraint decreases the maximum certainty equivalent of the liability holder from $C E_{L}(\Theta)=126.11$ to $C E_{L}(\Theta)=125.59$ (if $D_{0}=90$ ) and only slightly decreases the certainty equivalent from $C E_{L}(\Theta)=124.63$ to 124.58 if $D_{0}=94$.

We now want to carry out the same optimization under the flexible regulatory framework. Therefore, we set the initial regulatory barrier to $K_{0}=92$ (if $D_{0}=90$ ) and $K_{0}=95$ (if $D_{0}=94$ ). ${ }^{13}$ Then, we carry out the two optimizations over the parameter vector $\Theta=\left(\delta, \theta_{1}, \theta_{2}\right)$. In our numerical example, we first want to illustrate the optimization problem (17) without the 
default constraint. Therefore, Figure 2 varies the investment strategy $\left(\theta_{1}, \theta_{2}\right)$ fixing $L_{0}=95, D_{0}=90, K_{0}=92$. For each pair $\left(\theta_{1}, \theta_{2}\right)$, we then solve the optimization problem (17). The top graph presents the resulting value of the policyholder certainty equivalent $C E_{L}(\Theta)$, and the bottom graph the participation rate $\delta$ of this optimal contract. The white line represents the traditional regulatory framework, i.e. $\theta_{1}=\theta_{2}$. As can be observed from Figure 2, this white line does not contain the highest certainty equivalent $C E_{L}(\Theta)$, i.e. we can conclude that the flexible regulatory scheme leads to utility improvements to the liability holder. This highest certainty equivalent is given in Table 1 (bottom) together with the optimization results if a regulatory default constraint is imposed. This leads to several interesting observations. First, ignoring the regulator and its default constraint, the certainty equivalent increases slightly from 126.11 to 126.18 (if $D_{0}=90$ ) and from 124.63 to 125.23 (if $D_{0}=94$ ). Both results are slight improvements due to the higher flexibility in the investment strategy. If a default constraint is imposed, we first note from the bottom table that certainty equivalents are only very slightly (if $D_{0}=90$ ) or not at all (if $D_{0}=94$ ) affected (compare the results of optimization (17) and (18)). In the traditional regulatory framework presented in Table 1 (top), this effect is much stronger. This points to the advantage of the flexible regulatory framework where the asset strategy can be adapted to the default constraint - only marginally changing the utility for the policyholder. The resulting strategies in the flexible scheme typically lead to initially higher equity shares but a strong decrease in case of distress. Compared to the traditional regulatory framework, the certainty equivalent of the default constrained optimization (18) is significantly increased for both a default barrier $D_{0}=90$ and $D_{0}=94$.

\subsection{Insurance company is risk-averse}

In practice, the assumption of a complete market for insurance contracts is usually violated. Due to the long-term nature of the contracts, it is often not possible to fully hedge the inherent financial risk. In this case, the insurance company also values its payoff according to a utility function. Under this modification, we can confirm the results from Section 4.1. Details are given in the Appendix.

We have so far demonstrated that the change from the traditional to the flexible regulatory framework can significantly increase the maximal policyholder's utility - especially if a default constraint is imposed. That is why it is beneficial if the regulator enforces the optimal asset strategy that results from the flexible regulatory framework. The main reason why this new investment strategy leads to an improvement in policyholder utility is the fact that the flexible scheme allows for a higher upside potential (the initial equity share is higher than in the traditional scheme) while still keeping the default probabilities low (the equity share $\theta_{2}$ in distress is lower than in the traditional scheme). This leads to a higher maximal policyholder utility. If the default constraint is not binding, the maximal policyholder utility in the flexible regulatory framework also leads 

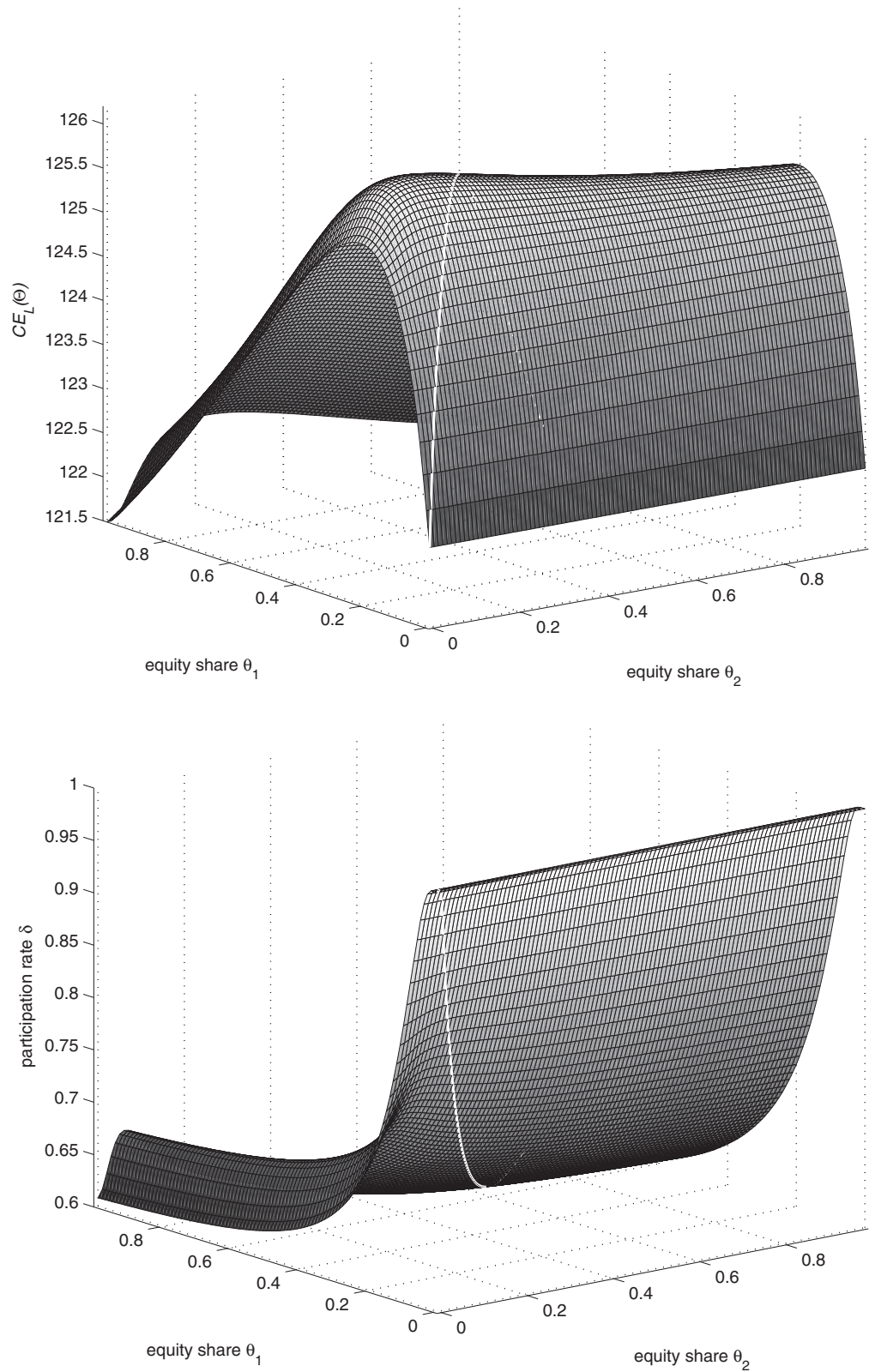

FIGURE 2: Certainty equivalent $C E_{L}(\Theta)$ of the liability holder (top) and fair participation rate $\delta$ (below) in a numerical example dependent on the investment strategy $\left(\theta_{1}, \theta_{2}\right)$. We set $K_{0}=92, A_{0}=100$,

$L_{0}=95, D_{0}=90, g=2.0 \%, T=10, \mu=6 \%, r=2.5 \%$ and $\sigma=0.2$. The contracts solve optimization problem (17) for a given strategy $\left(\theta_{1}, \theta_{2}\right)$. 
to a lower default probability than in the traditional regulatory framework (see Tables 1 and A1).

4.2.1. Discussion of assumptions. Now, we want to discuss some of our modeling assumptions and their implications on the results. One important and necessary assumption is the fact that the contract terms are agreed at the initiation of the contract and left unchanged until its maturity. Without this assumption, the insurance company might change the riskiness of the asset allocation contrasting the original agreement. This policy change might reduce the policyholder's utility or violate the regulatory default constraint. To avoid such a policy change the regulator's role is important. The regulator supervises the investment strategy, enforces its default constraint and intervenes as soon as the regulatory threshold is hit (yellow signal). Apart from this, a possibility to eliminate the equity holder's incentives for a change in the investment strategy is the addition of an incentive constraint " $\left(\delta, \theta_{1}, \theta_{2}\right) \in \operatorname{argmax} \mathcal{U}_{E}(\Theta)$ " in optimization (18) (see also Filipović et al. (2015)). Then, if the equity holder deviates from the initially agreed investment strategy, this would always deteriorate the equity holder's benefits. This extension is, however, numerically challenging and beyond the scope of this paper.

To focus on the effect of the different regulatory frameworks, we have introduced a rather simple insurance contract with only a terminal surplus participation. In practice, however, participating policies or collective defined contribution (DC) pension plans allow for periodic surplus participations. It is possible to extend our setup to a periodic surplus participation, however, the analytic tractability regarding the policyholder's utility $\mathcal{U}_{L}(\Theta)$ is lost. Intuitively, introducing periodic surplus participation without further modifications of parameters and model setup, would reduce the risk buffer and increase the default probability. If one optimizes $\Theta$ subject to a default constraint, this would result in a less-riskier investment strategy. The latter can be avoided if, for example, the surplus participation rate $\delta$ is lowered.

\section{CONCLUSION}

The present paper discusses flexible regulatory supervision that tries to lower the effect of a regulatory default constraint. Typically, the regulator imposes a Value-at-Risk-type constraint (default probability constraint) on the investment strategy. Such a constraint might force the insurance company to offer Pareto inefficient contracts with a lower utility of the policyholder. We show that this problem can be alleviated if the regulator enforces an optimal investment strategy that decreases risk in case of distress. Distress is defined by a "traffic light system" where the yellow traffic light (distress) is triggered as soon as the insurance company's asset value drops below a threshold $K$. The proposed strategy increases the policyholder's utility and leads to Pareto-improvements for the two involved parties. 
The advantage of this flexible scheme is that it is rather simple and - in contrast to, for example, a more flexible and dynamic asset allocation strategy like CPPI - easy to implement and supervise for a regulatory authority.

We want to stress that it can be beneficial to depart from the assumption of a constant volatility investment strategy that is frequently used in the literature. In further research, it is, for example, desirable to generalize our results to other dynamic asset allocation strategies.

\section{ACKNOWLEDGEMENTS}

Peter Hieber acknowledges funding by the German Association of Insurance Science (DVFVW). We want to thank Jing Li for fruitful discussions and her work on an earlier version of this manuscript. We are also thankful to two anonymous referees and for fruitful discussions at the IAA Colloquium in Oslo 2015, the World Risk and Insurance Economics Congress (WRIEC) 2015 in Munich, and the DMV meeting 2015 in Hamburg. An earlier version was entitled "Risk-shifting and optimal asset allocation in life insurance: The impact of regulation".

\section{NOTES}

1. See, e.g., §3(2) AnlV ("Anlageverordnung" = investment ordinance).

2. A contract is Pareto inefficient if a modification of contract terms could increase the benefits of at least one contract party without deteriorating the benefits of the other party.

3. In related areas such as asset-liability management, this has already been investigated. Here, some authors suggest that the riskiness of the asset investment should depend on the insurance company's funding ratio (=assets divided by liabilities). I.e. an insurance company should adapt its asset allocation depending on the possibility that it is (un)able to meet its obligations. Graf et al. (2012), Pézier and Scheller (2013) and Bohnert et al. (2015) suggest a CPPI-based strategy. Graf et al. (2011) and Hieber et al. (2015) change the asset allocation dependent on risk measures, i.e. the expected shortfall below the company's investment guarantees. Empirically, it is not obvious whether life insurance companies increase or decrease risk in case of distress: Mohan and Zhang (2014) find that US public funds increase risk if they are underfunded, while Rauh (2009) shows that the asset allocation is less risky if the company's financial condition is weaker.

4. We choose a relatively simple contract payoff. In reality, the policyholder is often entitled to a periodic surplus participation. However, since the main focus of this paper is to analyze diverse (rather) complex regulatory frameworks, we stick to this simple formulation of the contract payoff.

5. In case of the constant-mix investment strategy (2), we have that $\Theta=\left(\delta, \theta_{1}\right)$. Later on, the asset allocation strategy is allowed to be more flexible and, e.g., $\Theta=\left(\delta, \theta_{1}, \theta_{2}\right)$, see the following paragraph.

6. For a proof, see Equation (12) and footnote 9 in Section 3.

7. In practice, it is more reasonable to allow for a strategy recovery if assets $A_{t}$ return to values above the threshold $K_{t}$. It is possible to relax this assumption at the cost of additional complexity in the closed-form expressions provided in Section 3.

8. The event $\{\hat{\tau}>T\}$ delineates the situation that the assets perform well until maturity $T$ and all the time exceed the upper regulatory threshold. The event $\{\hat{\tau} \leq T, \tau>T\}$ describes the situation that the assets perform moderately until maturity $T$. The assets have hit the regulatory barrier but the insurance company has not defaulted prematurely. The event $\{\tau \leq T\}$ describes the situation that the company has defaulted before maturity $T$. 
9. A higher equity holding $\theta_{1}$ results in a higher probability of hitting the default barrier $D_{t}$. From Equation (12), we obtain

$$
\begin{aligned}
& \frac{\partial \mathbb{P}(\tau \leq T)}{\partial \theta_{1}}=\frac{-2 \ln \left(D_{0} / A_{0}\right)}{\sigma \theta_{1}^{2} \sqrt{T}} \varphi\left(\frac{\ln \left(D_{0} / A_{0}\right)-\tilde{\mu}_{1} T}{\sigma \theta_{1} \sqrt{T}}\right) \\
& \quad-\ln \left(D_{0} / A_{0}\right)\left(\frac{4(r-g) T}{\sigma^{2} \theta_{1}^{3}}+\frac{\mu-r}{\sigma^{2} \theta_{1}^{2}}\right)\left(\frac{D_{0}}{A_{0}}\right)^{\frac{2 \tilde{\mu}_{1}}{\sigma^{2} \theta_{1}^{2}}} \Phi\left(\frac{\ln \left(D_{0} / A_{0}\right)+\tilde{\mu}_{1} T}{\sigma \theta_{1} \sqrt{T}}\right)>0,
\end{aligned}
$$

since $D_{0}<A_{0}$ and $g \leq r<\mu$.

10. By Doob's optional sampling theorem, it holds that $A_{0}=\mathbb{E}_{\mathbb{Q}}\left[\mathbb{1}_{\{\tau>T\}} e^{-r T} A_{T}+\right.$ $\left.\mathbb{1}_{\{\tau \leq T\}} e^{-r \tau} A_{\tau}\right]=\mathbb{E}_{\mathbb{Q}}\left[e^{-r T}\left(V_{L}\left(A_{T}\right)+V_{E}\left(A_{T}\right)\right)\right]$. Thus, if Equation (15) is true, then Equation (16) also holds (and vice versa).

11. If we denote by $\lambda \geq 0$ the Lagrangian multiplier of the participation constraint, a derivation with respect to the participation rate leads to

$$
\frac{\partial \mathcal{U}_{L}(\Theta)}{\partial \delta}+\lambda \frac{\partial \mathbb{E}_{\mathbb{Q}}\left[e^{-r T} V_{E}\left(A_{T}\right)\right]}{\partial \delta}=0 .
$$

As $\partial \mathcal{U}_{L}(\Theta) / \partial \delta>0$ and $\partial \mathbb{E}_{\mathbb{Q}}\left[e^{-r T} V_{E}\left(A_{T}\right)\right] / \partial \delta<0, \lambda=0$ implies that the optimality criterion (19) cannot be satisfied. Therefore, only if $\lambda>0$, i.e. if the participation constraint is binding, we can achieve optimal solutions for problem (17). As default probability does not depend on the participation rate $\delta$ (see Equation (12)), this argument of a binding participation constraint also holds for Problem (18).

12. In order to interpret the results, we annualize default probabilities to $P D:=1-(1-\mathbb{P}(\tau \leq$ $T))^{1 / T}$. Note, however, that in our model framework, default times are not uniformly distributed over time (default probabilities for different time points can be obtained from Equations (12) and (13)). Thus, default probabilities cannot be summarized by just one number. The value presented can be seen as an average yearly default probability of the insurance company.

13. One could also include the parameter $K_{0}$ into the parameter vector $\Theta$ of the optimization. This, however, only very slightly affects the maximal certainty equivalent $C E_{L}(\Theta)$.

\section{REFERENCES}

BAuer, D., KIESEL, R., KLING, A. and Ruß, J. (2005) Risk neutral valuation of participating life insurance contracts. Insurance: Mathematics \& Economics, 39(2), 171-183.

BLACK, F. and COX, J.C. (1976) Valuing corporate securities: Some effects of bond indenture provisions. Journal of Finance, 31(2), 351-367.

Bohnert, A., GATZERT, N. and JøRGEnSEN, P. (2015) On the management of life insurance company risk by strategic choice of product mix, investment strategy and surplus appropriation schemes. Insurance: Mathematics \& Economics, 60, 83-97.

BRENNAN, M.J. and Schwartz, E.S. (1976) The pricing of equity-linked life insurance policies with an asset value guarantee. Journal of Financial Economics, 3(3), 195-213.

CHEN, A. and SUCHANECKI, M. (2007) Default risk, bankruptcy procedures and the market value of life insurance liabilities. Insurance: Mathematics and Economics, 40, 231-255.

DONG, M., GRÜNDL, H. and Schlütter, S. (2015) Is the risk-based meachanism always better? The risk-shifting behavior of insurers under different guarantee schemes. Journal of Insurance Issues, 38(1), 72-95.

Døskeland, T.M. and Nordahl, H.A. (2008) Optimal pension insurance design. Journal of Banking \& Finance, 32, 382-392.

European Parliament. (2014) Solvency II directive: Directive 2014/51/EU of the European Parliament and of the Council. Official Journal of the European Union, https://eiopa.europa.eu/ regulation-supervision/insurance/solvency-ii.

Filipović, D., KREMSleHner, R. and MuERMANN, A. (2015) Optimal investment and premium policies under risk shifting and solvency regulation. Journal of Risk and Insurance, 82(2), 261288. 
FolKs, J.L. and ChHIKARA, R.S. (1978) The inverse Gaussian distribution and its statistical application - a review. Journal of the Royal Statistical Society. Series B, 40(3), 263289.

GATZERT, N. and SCHMEISER, H. (2008) Combining fair pricing and capital requirements for nonlife insurance companies. Journal of Banking \& Finance, 32(12), 2589-2596.

GRAF, S., KLING, A. and RUß, J. (2011) Risk analysis and valuation of life insurance contracts: combining actuarial and financial approaches. Insurance: Mathematics \& Economics, 49(10), $115-125$.

Graf, S., Kling, A. and RUß, J. (2012) Financial planning and risk-return profiles. European Actuarial Journal 2, 77-104.

Grosen, J. and JøRGENSEN, P.L. (2002) Life insurance liabilities at market value: An analysis of insolvency risk, bonus policy, and regulatory intervention rules in a barrier option framework. Journal of Risk and Insurance, 69(1), 63-91.

He, H., Keirstead, W. and Rebholz, J. (1998) Double lookbacks. Mathematical Finance, 8(3), 201-228.

Hieber, P., Korn, R. and Scherer, M. (2015) Analyzing the effect of low interest rates on the surplus participation of life insurance policies with different annual interest rate guarantees. European Actuarial Journal, 5(1), 11-28.

JøRGENSEN, P.L. (2007) Traffic light options. Journal of Banking \& Finance, 31, 3698-3719.

MACMINN, R.D. and WITT, R.C. (1987) A financial theory of the insurance firm under uncertainty and regulatory constraints. Geneva Papers on Risk and Insurance, 12(42), 3-20.

MCCABE, G.M. and WITT, R.C. (1980) Insurance pricing and regulation under uncertainty: A chance-constrained approach. Journal of Risk and Insurance, 47(4), 607-635.

MoHAN, N. and ZHANG, T. (2014) An analysis of risk-taking behavior for public defined benefit plans. Journal of Banking \& Finance, 40, 403-419.

PÉZIER, J. and SCHELLER, J. (2013) Best portfolio insurance for long-term investment strategies in realistic conditions. Insurance: Mathematics \& Economics, 52, 263-274.

RAUH, J.D. (2009) Risk shifting versus risk management: Investment policy in corporate pension plans. Review of Financial Studies, 22(7), 2687-2733.

ReEs, R., Gravelle, H. and WAmbach, A. (1999) Regulation of insurance markets. Geneva Papers on Risk and Insurance Theory, 24, 55-68.

SCHLÜTTER, S. (2014) Capital requirements or pricing constraints? An economic analysis of measures for insurance regulation. Journal of Risk Finance, 15(5), 533-554.

SCHMEISER, H. and WAGNER, J. (2013) The impact of introducing insurance guaranty schemes on pricing and capital structure. Journal of Risk and Insurance, 80(2), 273-308.

SCHMEISER, H. and WAGNER, J. (2015) A proposal on how the regulator should set minimum interest rate guarantees in participating life insurance contracts. Journal of Risk and Insurance, 82(3), 659-686.

SHREVE, S. (2004) Stochastic Calculus for Finance II. New York: Springer.

\title{
AN CHEN
}

\author{
Institute of Insurance Science \\ University of Ulm \\ Helmholtzstr. 20 \\ 89069 Ulm, Germany \\ E-Mail:an.chen@uni-ulm.de
}

\author{
Peter Hieber (Corresponding author) \\ Institute of Insurance Science \\ University of Ulm \\ Helmholtzstr. 20 \\ 89069 Ulm, Germany \\ E-Mail:peter.hieber@uni-ulm.de
}




\section{APPENDIX A}

Proof of Theorem 3.1. First, we recall results on the first-hitting time $\tau$ of a geometric Brownian motion, i.e. the process $A$ as defined in (2). The law of $\tau$ is known to be inverse Gaussian (see, e.g., Folks and Chhikara (1978)). Lemma A.1 recalls some results on the firsthitting time in this special case.

Lemma A.1 (First-hitting time distribution). Consider the process A from (2). Then, the survival probability within the interval $(t, T]$ is given by

$$
\mathbb{P}(\tau>T \mid \tau>t)=\Phi\left(\frac{\tilde{\mu}_{1}(T-t)-\ln \left(D_{t} / A_{t}\right)}{\sigma \theta_{1} \sqrt{T-t}}\right)-\left(\frac{D_{t}}{A_{t}}\right)^{\frac{2 \tilde{\mu}_{1}}{\sigma^{2} \theta_{1}^{2}}} \Phi\left(\frac{\tilde{\mu}_{1}(T-t)+\ln \left(D_{t} / A_{t}\right)}{\sigma \theta_{1} \sqrt{T-t}}\right),
$$

where $D_{t}<A_{t}, \tilde{\mu}_{1}:=r+\theta_{1}(\mu-r)-g-\sigma^{2} \theta_{1}^{2} / 2$, and $\Phi(\cdot)$ denotes the standard normal cumulative distribution function. The density of $\tau$ can be obtained from

$$
f^{(i)}\left(t, \tau, A_{t}, D_{t}\right):=\frac{-\ln \left(D_{t} / A_{t}\right)}{\sigma \theta_{i}(\tau-t)^{\frac{3}{2}}} \varphi\left(\frac{\ln \left(D_{t} / A_{t}\right)-\tilde{\mu}_{i}(\tau-t)}{\sigma \theta_{i} \sqrt{\tau-t}}\right) .
$$

For $y:=\ln \left(e^{-g T} A_{T} / A_{t}\right)$, we define $g^{(1)}\left(y, t, T, A_{t}, D_{t}\right):=\mathbb{P}(y \in d y, \tau>T)$, which is known to be

$$
g^{(1)}\left(y, t, T, A_{t}, D_{t}\right)=\left\{\begin{array}{ll}
0 & \text { for } y \leq \ln \left(D_{t} / A_{t}\right) \\
\frac{\varphi\left(\frac{y-\tilde{\mu}_{i}(T-t)}{\sigma \theta_{1} \sqrt{T-t}}\right)}{\sigma \theta_{1} \sqrt{T-t}}\left(1-e^{\left.-2 \frac{\ln \left(D_{t} / A_{t}\right)^{2}-y \ln \left(D_{t} / A_{t}\right)}{\sigma^{2} \theta_{1}^{2}(T-t)}\right)}\right. & \text { else }
\end{array},\right.
$$

where $\varphi(\cdot)$ denotes the density of the standard normal distribution.

Proof. See, e.g., Folks and Chhikara (1978); He et al. (1998); Shreve (2004).

Note that the same results hold, if we replace the asset strategy $\theta_{1}$ by $\theta_{2}$ (and similarly $\tilde{\mu}_{1}$ by $\left.\tilde{\mu}_{2}:=r+\theta_{2}(\mu-r)-g-\sigma^{2} \theta_{2}^{2} / 2\right)$. We denote the densities that result from this parameter change by $f^{(2)}\left(t, \tau, A_{\tau}, D_{\tau}\right)$, respectively $g^{(2)}\left(y, t, T, A_{t}, D_{t}\right)$.

We now use Lemma A.1 to prove Theorem 3.1. Note first that if the barrier $D$ is not hit in the interval $(0, T]$, (A2) helps us to obtain the distribution of the assets $A$ at maturity $T$. To compute the expected utility of the terminal payoff $\Psi_{L}\left(A_{T}\right)$ from (4), one simply has to integrate its utility over (A2) on the set $\left(\ln \left(e^{-g T} D_{T} / A_{0}\right), \infty\right)=\left(\ln \left(D_{0} / A_{0}\right), \infty\right)$. If the barrier is hit, i.e. $\tau \leq T$, the terminal payoff depends solely on the default time $\tau$ whose distribution can be obtained from (A1). This then leads to

$$
\begin{aligned}
\mathbb{E}_{\mathbb{P}}\left[u_{L}\left(V_{L}\left(A_{T}\right)\right)\right]= & \mathbb{E}_{\mathbb{P}}\left[\mathbb{1}_{\{\tau>T\}} u_{L}\left(\Psi_{L}\left(A_{T}\right)\right)\right]+\mathbb{E}_{\mathbb{P}}\left[\mathbb{1}_{\{\tau \leq T\}} u_{L}\left(e^{r(T-\tau)} \Xi_{L}(\tau)\right)\right] \\
= & \int_{\ln \left(D_{0} / A_{0}\right)}^{\infty} u_{L}\left(\Psi_{L}\left(A_{0} e^{y+g T}\right)\right) \cdot g^{(1)}\left(y, 0, T, A_{t}, D_{t}\right) \mathrm{d} y \\
& +\int_{0}^{T} u_{L}\left(e^{r T} \Xi_{L}(\tau)\right) \cdot f^{(1)}\left(0, \tau, A_{0}, D_{0}\right) \mathrm{d} \tau .
\end{aligned}
$$


In the case of power utility (see Example 2.2), the latter integrals can be further simplified.

Proof of Theorem 3.2. Theorem 3.2 can be derived using the previous results. Note that the regulatory barrier $K_{t}=K_{0} e^{g t}$ is always hit prior to default due to the continuity of the process $A$. Up to time $\hat{\tau}$ the process $A$ is a geometric Brownian motion with strategy $\theta_{1}$ allowing us to use the density $f^{(1)}\left(t, \hat{\tau}, A_{t}, K_{t}\right)$ from Lemma A. 1 for $\hat{\tau}$. At time $\hat{\tau}$, we are back in the situation that is already solved in Theorem 3.1: One has to adapt the initial values for $A, D$, and $L$. Furthermore, the time to maturity is now $T-\hat{\tau}$ instead of $T$ and the investment strategy is now $\theta_{2}$. If the regulatory threshold $K$ is never hit, we can in analogy to the proof of Theorem 3.1 compute the expected utility of the terminal payoffs to get the first terms of $\zeta_{L}\left(A_{0}, D_{0}, K_{0}, L_{0}, T\right)$ :

$$
\begin{aligned}
\zeta_{L}\left(A_{0}, D_{0}, K_{0}, L_{0}, T\right)= & \int_{0}^{T} \kappa_{L}^{(2)}\left(K_{\hat{\tau}}, D_{\hat{\tau}}, L_{\hat{\tau}}, \hat{\tau}, T\right) \cdot f^{(1)}\left(0, \hat{\tau}, A_{0}, K_{0}\right) \mathrm{d} \hat{\tau} \\
& +\int_{\ln \left(K_{0} / A_{0}\right)}^{\infty} u_{L}\left(\Psi_{L}\left(A_{0} e^{y+g T}\right)\right) g^{(1)}\left(y, 0, T, A_{0}, K_{0}\right) \mathrm{d} y,
\end{aligned}
$$

an expression that can be rewritten to

$$
\begin{aligned}
= & \int_{0}^{T} \int_{\ln \left(D_{0} / K_{0}\right)}^{\infty} u_{L}\left(\Psi_{L}\left(K_{\hat{\tau}} e^{y+g(T-\hat{\tau})}\right) \cdot f^{(1)}\left(0, \hat{\tau}, A_{0}, K_{0}\right) \cdot g^{(2)}\left(y, \hat{\tau}, T, K_{\hat{\tau}}, D_{\hat{\tau}}\right) \mathrm{d} y \mathrm{~d} \hat{\tau}\right. \\
& +\int_{0}^{T} \int_{\hat{\tau}}^{T} u_{L}\left(e^{r(T-\tau)} \Xi_{L}(\tau)\right) \cdot f^{(1)}\left(0, \hat{\tau}, A_{0}, K_{0}\right) \cdot f^{(2)}\left(\hat{\tau}, \tau, K_{\hat{\tau}}, D_{\hat{\tau}}\right) \mathrm{d} \tau \mathrm{d} \hat{\tau} \\
& +\int_{\ln \left(K_{0} / A_{0}\right)}^{\infty} u_{L}\left(\Xi_{L}\left(A_{0} e^{y+g T}\right)\right) \cdot g^{(1)}\left(y, 0, T, A_{0}, K_{0}\right) \mathrm{d} y,
\end{aligned}
$$

with $\kappa_{L}^{(2)}(\cdot), f$, and $g$ as defined in Theorem 3.1. Again, power utility simplifies the given expressions.

Section 4.2: Insurance company is risk-averse. In contrast to the fair pricing constraint in Section 4.1, we now assume that the insurance company is risk-averse and thus values its payoff according to a utility function, i.e.

$$
\mathcal{U}_{E}(\Theta):=\mathbb{E}_{\mathbb{P}}\left[u_{E}\left(V_{E}\left(A_{T}\right)\right)\right]
$$

This expectation can also be computed using the results in Theorems 3.1 and 3.2, respectively, if one replaces the index $L$ by $E$ (i.e. the payoffs $\Xi_{L}(\tau), \Psi_{L}\left(A_{T}\right)$ by $\Xi_{E}(\tau), \Psi_{E}\left(A_{T}\right)$, the utility function $u_{L}(x)$ by $u_{E}(x)$, etc.). In analogy to Section 4.1 , we define a participation constraint for the equity holder. Insurance contracts are initiated if the utility $\mathcal{U}_{E}(\Theta)$ exceeds some minimum reservation utility $U_{0} \in \mathbb{R}$. This leads to the regulatory optimization problem, again maximizing the policyholder's utility subject to a participation constraint:

$$
\begin{aligned}
& \max _{\Theta} \mathcal{U}_{L}(\Theta) \\
& \text { s.t. } U_{0} \leq \mathcal{U}_{E}(\Theta),
\end{aligned}
$$

where $\Theta=\left(\delta, \theta_{1}\right)$ in the traditional regulatory framework and $\Theta=\left(\delta, \theta_{1}, \theta_{2}\right)$ under the flexible regulatory scheme. If, additionally, the regulator imposes a default constraint, the 
TABLE A1

RESULTS OF THE UTILITY MAXIMIZATION PROBLEMS (A5) (NO DEFAULT CONSTRAINT) AND (A6) (DEFAULT CONSTRAINT $P D \leq 0.5 \%$ ) IN THE TRADITIONAL AND FLEXIBLE REGULATORY FRAMEWORK. THE ANNUALIZED DEFAULT PROBABILITY $P D$ IS DEFINED AS $P D:=1-(1-\mathbb{P}(\tau \leq T))^{1 / T}$.

\begin{tabular}{|c|c|c|c|c|c|}
\hline \multicolumn{6}{|c|}{ Traditional Regulatory Framework } \\
\hline & & $C E_{L}(\Theta)$ & $\theta_{1}^{*}$ & $\delta^{*}$ & $P D$ \\
\hline \multirow{2}{*}{$L_{0}=95, D_{0}=90$} & no default constraint, (A5) & 128.27 & $26.3 \%$ & $89.4 \%$ & $2.56 \%$ \\
\hline & default constraint, (A6) & 127.07 & $14.3 \%$ & $96.6 \%$ & $0.50 \%$ \\
\hline \multirow{3}{*}{$L_{0}=95, D_{0}=94$} & onstraint, (A5) & 126.91 & $22.2 \%$ & $78.1 \%$ & $3.50 \%$ \\
\hline & default constraint, (A6) & 125.65 & $9.7 \%$ & $97.1 \%$ & $0.50 \%$ \\
\hline & \multicolumn{5}{|c|}{ Flexible Regulatory Framework } \\
\hline & & $C E_{L}(\Theta)$ & $\left(\theta_{1}^{*}, \theta_{2}^{*}\right)$ & $\delta^{*}$ & $P D$ \\
\hline \multirow{2}{*}{\multicolumn{2}{|c|}{$\begin{array}{r}L_{0}=95, D_{0}=90, K_{0}=92 \text { no default constraint, (A5) } \\
\text { default constraint, (A6) }\end{array}$}} & 128.35 & $7.6 \%, 15.5 \%)$ & $90.6 \%$ & $2.12 \%$ \\
\hline & & 128.19 & $25.8 \%, 6.6 \%)$ & $92.4 \%$ & $0.50 \%$ \\
\hline \multirow{2}{*}{\multicolumn{2}{|c|}{$\begin{array}{r}L_{0}=95, D_{0}=94, K_{0}=95 \text { no default constraint, (A5) } \\
\text { default constraint, (A6) }\end{array}$}} & 127.00 & $3.5 \%, 11.1 \%)$ & $82.2 \%$ & $2.95 \%$ \\
\hline & & 126.72 & $19.4 \%, 4$ & $95.3 \%$ & $0.50 \%$ \\
\hline
\end{tabular}

optimization problem has to be modified to

$$
\begin{array}{ll}
\max _{\Theta} & \mathcal{U}_{L}(\Theta) \\
\text { s.t. } & U_{0} \leq \mathcal{U}_{E}(\Theta) \\
& \mathbb{P}(\tau \leq T) \leq \epsilon,
\end{array}
$$

where, again, $\epsilon>0$ is the upper bound on the company's default probability. Also here, problems (A5) and (A6) have to be solved numerically. For the optimal solution, the participation constraint is again binding.

Numerical illustrations. We use the same parameter set as in Section 4.1. The utility function of the insurance company is assumed to be power utility $u_{E}\left(V_{E}\right):=V_{E}^{1-\gamma_{E}} /\left(1-\gamma_{E}\right)$ with a relative risk-aversion parameter of $0<\gamma_{E}=0.2<1<\gamma_{L}$. It is reasonable to assume that the equity holder is less risk-averse than the liability holder. Table A1 illustrates the optimal results from the utility maximization problems (A5) and (A6). In this example, we take a risk-free investment as the reference investment and set the reservation utility to $U_{0}=\left(E_{0} e^{r T}\right)^{1-\gamma_{E}} /\left(1-\gamma_{E}\right)$. Participating in the insurance contracts leads to a utility level which is at least as high as investing in the risk-free asset.

The results in Table A1 are very similar to Table 1 in Section 4.1. From the perspective of optimal asset allocation (no default constraint), the introduction of the flexible regulatory scheme leads to only small changes in the maximal policyholder utility $\left(C E_{L}(\Theta)=128.27\right.$ to 128.35 if $D_{0}=94$ and $C E_{L}(\Theta)=126.91$ to 127.00 if $\left.D_{0}=90\right)$. Interesting is the case where an additional default constraint is imposed by the regulator. In the traditional regulatory framework, a default constraint leads to a significant drop in the maximal policyholder certainty equivalent (compare the result of optimizations (A5) and (A6) in Table A1). In contrast, the resulting maximal certainty equivalent $C E_{L}(\Theta)$ in the flexible regulatory framework is only slightly affected by a default constraint. The optimal flexible strategy is again a 


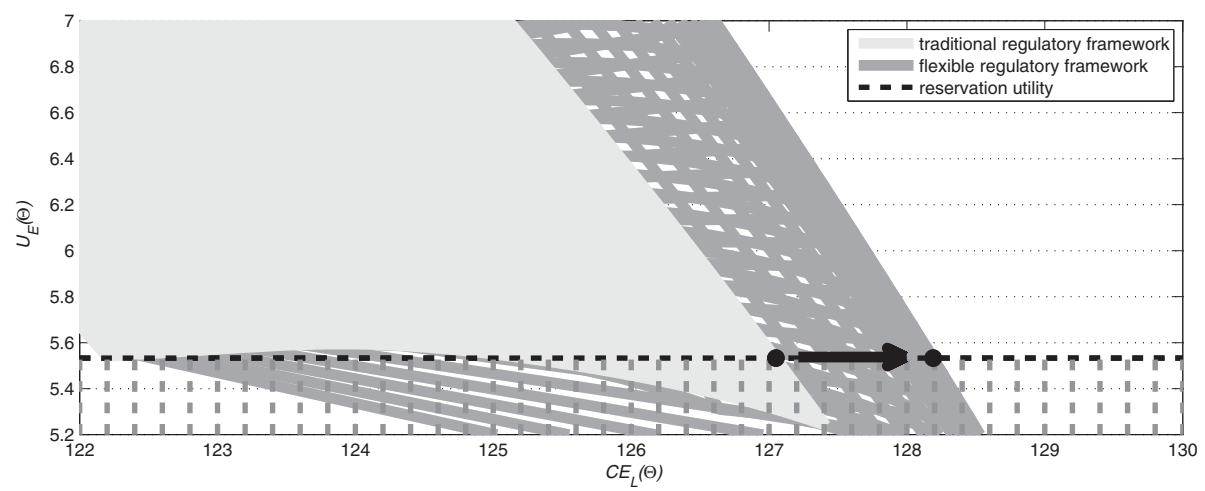

FIGURE 3: Objective function pairs $\left(\mathcal{U}_{L}(\Theta), \mathcal{U}_{E}(\Theta)\right)$ in case of a default constraint. Each point in this graph corresponds to one possible insurance contract with an annualized default probability $P D:=1-(1-\mathbb{P}(\tau \leq T))^{1 / T}$ smaller than $0.5 \%$ and parameter vector $\left(\delta, \theta_{1}, \theta_{2}\right)$.

reduction of the riskiness of the investment strategy in distress $\left(\theta_{2}<\theta_{1}\right)$. In order to demonstrate the advantages of adopting our flexible regulation framework, we re-illustrate the first example from Table $1\left(L_{0}=95, D_{0}=90, K_{0}=92\right.$, default constraint) in Figure 3. For different values of $\Theta=\left(\delta, \theta_{1}\right)$ (traditional regulatory framework) and $\Theta=\left(\delta, \theta_{1}, \theta_{2}\right)$ (flexible regulatory framework), one can compute the utility of the policyholder (see Equation (8)) and the equity holder (see Equation (A4)). Each parameter set can then be represented as a utility pair $\left(\mathcal{U}_{L}(\Theta), \mathcal{U}_{E}(\Theta)\right)$. The light gray area represents combinations that can be reached by the traditional regulatory framework, the dark (and light) gray area those that can be reached by the flexible regulatory framework. The black-dashed line represents the reservation utility $U_{0}$ - each contract lying above (or on) this line fulfills the participation constraint. The two black dots on this line represent the contracts with maximal policyholder utility under the additional default constraint, i.e. the solutions $C E_{L}(\Theta)=127.07$ (traditional regulatory scheme) and $C E_{L}(\Theta)=128.19$ (flexible regulatory scheme) of optimization (A6), see Table A1. The black arrow demonstrates the improvement in utility of the liability holder by introducing the flexible regulatory scheme. Obviously, for each contract term in the traditional framework, we can find one contract in the flexible regulatory framework that has a higher utility for the policyholder, keeping the equity holder's reservation utility constant. More formally, one can state that the flexible regulatory scheme Pareto-dominates the traditional regulatory scheme, see Definition A.2.

Definition A.2 (Pareto-efficient contract terms). Contract terms $\Theta^{*}=\left(\delta, \theta_{1}, \theta_{2}\right) \in \mathcal{X}$ are Pareto-efficient if there does not exist a contract term $\Theta \in \mathcal{X}$ such that

$$
\Theta \succ \Theta^{*},
$$

meaning that at least one of the following holds:

$$
\begin{aligned}
& \mathcal{U}_{L}(\Theta) \geq \mathcal{U}_{L}\left(\Theta^{*}\right) \text { and } \mathcal{U}_{E}(\Theta)>\mathcal{U}_{E}\left(\Theta^{*}\right), \quad \text { or } \\
& \mathcal{U}_{L}(\Theta)>\mathcal{U}_{L}\left(\Theta^{*}\right) \text { and } \mathcal{U}_{E}(\Theta) \geq \mathcal{U}_{E}\left(\Theta^{*}\right) .
\end{aligned}
$$

